\title{
Scanning direction and line bisection: a study of normal subjects and unilateral neglect patients with opposite reading habits
}

\author{
Sylvie Chokron ${ }^{\mathrm{a}, \mathrm{b}, *}$, Paolo Bartolomeo ${ }^{\mathrm{c}, \mathrm{d}}$, Marie-Thérèse Perenin ${ }^{\mathrm{e}}$, Gérard Helft ${ }^{\mathrm{f}}$, \\ Michel Imbert ${ }^{\mathrm{g}}$ \\ a Laboratoire de Psychologie Expérimentale, CNRS ep 617, Université de Savoie, Chambéry, France \\ ${ }^{\mathrm{b}}$ Service de Neurologie, Fondation Ophtalmologique Rothschild, 25 rue Manin, 75019 Paris, France \\ c INSERM Unit 324, Paris, France \\ ${ }^{\mathrm{d}}$ Neuroscience Department, Henri-Mondor Hospital, Créteil, France

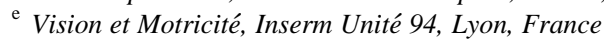 \\ ${ }^{\mathrm{f}}$ Hôpital Necker, Paris, France \\ ${ }^{\mathrm{g}}$ Unité Cerveau et Cognition, CNRS 9940, Toulouse, France
}

Accepted 19 May 1998

\begin{abstract}
Sixty normal dextrals (30 left-to-right and 30 right-to-left readers) and two left unilateral neglect patient with opposite reading habits performed a passive line bisection task. In order to study the effect of scanning direction on performance, subjects had to stop a mark moving on the to-be-bisected line either from the left to the right or in the opposite direction. Results showed that the position of the subjective middle was dependent upon the scanning direction of the line for all the subjects. A leftward deviation appeared for left to right scanning, whereas a rightward shift occurred when the mark moved from the right to the left. These results emphasize the role of scanning direction in space organization and are discussed with respect to the explanatory hypotheses of unilateral neglect. (C) 1998 Elsevier Science B.V. All rights reserved.
\end{abstract}

Keywords: Line bisection; Neglect syndrome; Normal subject; Reading habit; Scanning direction; Left-to-right reader; Right-to-left reader

\section{Introduction}

Unilateral neglect is a neurological disorder clinically characterized by the inability to perceive or orient to stimuli presented to one side of space (usually the left), despite the absence of significant sensory or motor deficit $[14,21]$. Typically, when asked to indicate the middle of a visually perceived line (i.e., visuo-motor bisection task), right brain-damaged patients with left neglect tend to transect the line to the right of the geometric midpoint [34]. Certain studies have emphasized the role of exploration strategies on left neglect $[29,36,37]$. Weintraub and Mesulam [36], suggest that damage to the right hemisphere (but not to the left), impairs search strategies and this may contribute to the severity of the visual inattention observed in patients with this kind of lesion. Their study revealed that patients with left sided lesions like normal control

\footnotetext{
* Corresponding author. Fax: +33-01-48-03-68-59; E-mail: chokron@ext.jussieu.fr
}

subjects initiated the search on the left side of the page and spontaneously adopted a systematic search pattern even with an unstructured array. In contrast, patients with right cerebral lesions typically began on the right side of the page and searched erratically, particularly when the stimulus array was unstructured (see also Refs. $[2,16]$ ). The 'right bias' observed in bisection tasks among left-neglect patients, may be reduced by visual $[30,33]$ or spatio-motor cueing $[17,31]$ but also by manipulating scanning direction.

Reuter-Lorenz and Posner [29], showed that the rightward transection observed among right-damaged patients with neglect decreased in a passive visuo-motor bisection task, when the experimentor moved a pen along the line from left to right as opposed to the right-to-left condition, which increased the amount of rightward shift. This effect of scanning direction on passive line bisection was replicated by Mattingley et al. [24] among normal subjects and left neglect patients.

In the same way, the study of Halligan and Marshall [20] revealed an effect of the starting position and in this 
way of the scanning direction on line bisection performed with computerised visual display unit.

In fact, Halligan et al. [18] maintain that bisection is dependent upon the 'attentional' direction to approach the midpoint; they hypothesize that left neglect patients approach the midpoint from the right and stop short of the true midpoint by a value corresponding to their (pathologically increased) Weber fraction.

The use of bisection protocols with normal subjects has made obvious an asymmetric perception of space, with a tendency to place the subjective middle to the left of the objective middle when the subject is asked to estimate the centre of a line $[6,7,23,26]$ or a rod $[5-7,11,32]$. The position of the subjective middle during visuo-motor or tactilo-kinesthetic bisection tasks seems sensitive to various parameters, including the laterality of the subject [32], the hand used [11,32], gravitational coordinates [6] and the direction of gaze [11].

Two recent studies $[10,12]$ demonstrated that the estimation of the subjective middle during a classical visuomotor task may depend on the subject's reading habits. Left-to-right readers (French normal subjects) deviated to the left of the objective middle, whereas right-to-left readers (Israeli subjects) deviated to the right. This effect of reading habits on normal subjects' performance was also found in a line extension task, where the subject had to construct either the right half of a line from the left given half line or the reverse [9]. Whereas left-to-right readers significantly underconstructed the left half from the right given one, right-to-left readers exhibited no significant bias during this task. Interestingly, when two left neglect patients with opposite reading habits were studied with the same paradigm, they did not show a different pattern from each other, both underconstructing the right half from the left given half and overconstructing the left half from the right given one [9], thus confirming previous results in that they reproduced the usual rightward bias in the line extension task $[3,4]$. Note also that, in a straight-ahead pointing task, the position of the subjective straight ahead was dependent upon the motor direction in both normal and neglect subjects $[8,13]$.

Thus, these findings supported the idea that acquired directional trends like reading habits may affect subjects' performance in visuo-spatial tasks [1].

The aim of the present study was to investigate how an imposed scanning direction can play a role on space perception among normal dextrals and neglect patients with opposite reading habits. In other words, is it possible to change the perception of the subjective middle of a normal subject by changing the scanning direction of the line? We employed a passive visuo-motor bisection task, with exploration of the line from left to right or from right to left in 60 normal dextrals with opposite reading habits (30 left-to-right readers and 30 right-to-left readers) and in two left unilateral neglect patients with opposite reading habits.
On the basis of the previously reviewed findings, we expected that an imposed scanning direction from left to right or from right to left may have had an effect on the perception of the subjective middle by normal and neglect subjects.

\section{Method}

\subsection{Subjects}

Thirty right handed normal French subjects reading from left-to-right ( 8 men, 22 women), average age 22.8 (from 18.5 to 29.3) and 30 normal Israeli dextrals (15 women, 15 men, ranging from 20.7 to 28.3 years with an average age of 23.2 years) volunteers to participate in the study. None of the subjects was bilingual. Laterality was assessed by using the Delatollas et al.'s questionnaire [15].

Two left neglect patients exempt from visual fields defect consented to participate in this study. Both had lesions involving the right parietal lobe.

Patient 1 was a 70-year-old French man, left-to-right reader, who had undergone a vascular infarct in the right middle cerebral artery area 2 months before. CT scan showed a lesion centered on the right parieto-occipital carrefour. He was affected by a severe left hemiplegia and somatosensory deficit.

Patient 2 was a 46-year-old Israeli man, right-to-left reader, suffering from left unilateral neglect after a right parietal lesion consecutive to a CVA 4 months before experimental testing.

CT-scan showed a right temporo-parietal lesion. Both patients had; left neglect was assessed through a series of tasks, including crossing-out line segments, bisection, drawing and writing.

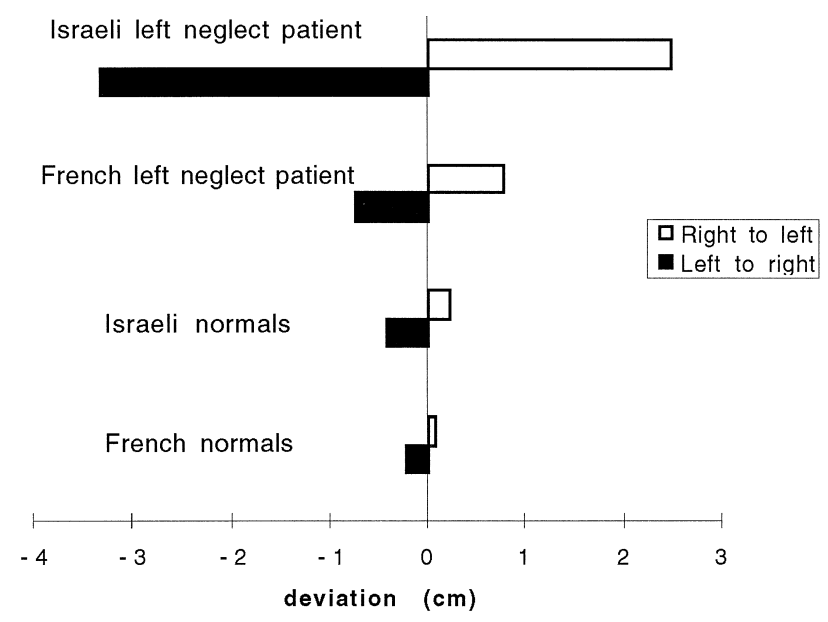

Fig. 1. Effect of scanning direction on deviation in line bisection. 
Table 1

Passive visuo-motor bisection and scanning direction: normal subjects $(n=60)$. Constant errors $(\mathrm{cm}) ; \pm 1$ standard deviation

\begin{tabular}{|c|c|c|c|c|c|c|c|c|}
\hline & \multicolumn{4}{|c|}{ Left-to-right scanning } & \multicolumn{4}{|c|}{ Right-to-left scanning } \\
\hline & $5 \mathrm{~cm}$ & $15 \mathrm{~cm}$ & $20 \mathrm{~cm}$ & Mean & $5 \mathrm{~cm}$ & $15 \mathrm{~cm}$ & $20 \mathrm{~cm}$ & Mean \\
\hline $\begin{array}{l}\text { French normals } \\
\text { Israeli normals }\end{array}$ & $\begin{array}{l}-0.13( \pm 0.17) \\
-0.20( \pm 0.11)\end{array}$ & $\begin{array}{l}-0.24( \pm 0.28) \\
-0.46( \pm 0.27)\end{array}$ & $\begin{array}{l}-0.30( \pm 0.31) \\
-0.62( \pm 0.36)\end{array}$ & $\begin{array}{l}-0.22( \pm 0.22) \\
-0.43( \pm 0.21)\end{array}$ & $\begin{array}{l}+0.04( \pm 0.12) \\
+0.19( \pm 0.10)\end{array}$ & $\begin{array}{l}+1.49( \pm 0.26) \\
+2.86( \pm 0.30)\end{array}$ & $\begin{array}{r}+1.18( \pm 0.23) \\
3.23( \pm 0.37)\end{array}$ & $\begin{array}{l}+0.77( \pm 0.27) \\
+2.48( \pm 0.34)\end{array}$ \\
\hline
\end{tabular}

-: Leftward deviation; + : rightward deviation.

Table 2

Passive visuo-motor bisection and scanning direction: French and Israeli Left neglect patients $(n=2)$. Constant errors $(\mathrm{cm}) \pm 1$ standard deviation

\begin{tabular}{|c|c|c|c|c|c|c|c|c|}
\hline & \multicolumn{4}{|c|}{ Left-to-right scanning } & \multicolumn{4}{|c|}{ Right-to-left scanning } \\
\hline & $5 \mathrm{~cm}$ & $15 \mathrm{~cm}$ & $20 \mathrm{~cm}$ & Mean & $5 \mathrm{~cm}$ & $15 \mathrm{~cm}$ & $20 \mathrm{~cm}$ & Mean \\
\hline French left neglect patient & $-0.90( \pm 0.32)$ & $-0.49( \pm 0.31)$ & $-0.88( \pm 0.31)$ & $-0.75( \pm 0.35)$ & $+0.04( \pm 0.21)$ & $+0.03( \pm 0.26)$ & $+0.13( \pm 0.23)$ & $+0.07(0.16)$ \\
\hline Israeli left neglect patient & $-1.31( \pm 0.19)$ & $-4.29( \pm 0.29)$ & $-4.43( \pm 0.46)$ & $-3.34( \pm 0.43)$ & $+1.35( \pm 0.16)$ & $+0.21( \pm 0.17)$ & $+0.24( \pm 0.26)$ & $+0.22( \pm 0.15)$ \\
\hline
\end{tabular}

- : Leftward deviation; + : rightward deviation. 


\subsection{Stimuli}

One-millimeter wide horizontal lines were presented on a computer screen. Three line lengths were used: 5, 15 and $20 \mathrm{~cm}$. Eight samples were presented for each line length. The lines were centered horizontally and vertically on the screen.

\subsection{Procedure}

The subject was seated at $57 \mathrm{~cm}$ from the screen, his sagittal middle corresponding to the midsection of the screen. Each line appeared individually on the screen, with a 1-cm vertical mark either at its left or at its right end. The subject was told that the mark could move from one extremity to the other and that he or she had to stop it as soon as it crossed the middle of the line. The displacement of the mark was about $2 \mathrm{~cm} / \mathrm{s}$ so as not to restrict the visual exploration (same speed as in Ref. [24]). If the subject had not stopped the mark when it had reached the end of the line, the mark went back automatically to the starting point, at the left end for left-to-right scan, or at the right end for right-to-left scan. The task was to start the displacement by pressing a key and to stop it by pressing the same key at the point estimated by the subject as being the middle. All subjects used their right hand. One block of 24 trials for each scanning direction was administered. The order of presentation of the blocks was counterbalanced among the subjects. The order of presentation of lines was randomized as concerns line length.

The errors were noted to the nearest millimetre and carried a plus if the bisection was to the right of the objective middle and a minus if the bisection was to the left of the objective middle.

\section{Results}

Fig. 1 reports mean deviations for all subjects and conditions.

\subsection{Normal french subjects}

A repeated measures ANOVA with sex, scanning direction, and length line as factors revealed a significant effect of the scanning direction on the position of the subjective middle $\left(F_{1-27}=43,38 ; p<0.005\right)$. In fact, subjects transected the line to the left of the objective middle when scanned from left to right $\left(m=-0.22 \mathrm{~cm} ; t_{29}=5.47\right.$; $p<0.005$ ), and to the right of the objective middle when scanning from right to left $\left(m=+0.07 ; t_{29}=2.39 ; p<\right.$ 0.05 ) (Table 1). An interaction effect between length and scanning direction occurred $\left(F_{2-54}=8,81 ; p<0.005\right)$, in the form of an increased error for longer lines, with leftward deviation for left to right scan and rightward deviation for right to left scan (Table 1).

\subsection{Normal israeli subjects}

A repeated measures ANOVA with sex, scanning direction, and length line as factors revealed a significant effect of the scanning direction on the position of the subjective middle $\left(F_{1-27}=19,7 ; p<0.005\right)$. Again, left-to-right scanning revealed a significant leftward deviation of the subjective middle $\left(m=-0.43 \mathrm{~cm} ; t_{29}=; p<0.005\right)$, while right-to-left scanning entailed a significant rightward deviation $\left(m=+0.21 ; t_{29}=7.9 ; p<0.05\right)$ (Table 1$)$.

\subsection{French left neglect patient}

Scanning the line from right to left induced a rightward bias $(m=+0.77 \mathrm{~cm})$ in this patient whilst scanning from left to right reversed the deviation, i.e., induced a leftward deviation $(m=-0.75 \mathrm{~cm})$, (see Table 2$)$. Separate analysis for each line length revealed that the shortest line (5 $\mathrm{cm}$ ) was always bisected to the left of the objective middle (Table 2), whatever the scanning direction. For the other lines $(15$ and $20 \mathrm{~cm}$ ), the leftward deviation for left-to-right scanning and the rightward deviation for right-to-left scanning, increased with the line length (Table 2).

\subsection{Israeli left neglect patient}

When scanning from right to left, the usual rightward deviation is recorded $(m=+2.48)$ (table). By contrast, scanning the line from left-to-right produced a reversed pattern with a strong leftward deviation $(m=-3.34)$ (Table 2).

For left-to-right scanning, the longer was the line the more leftward was the deviation of the subjective middle; similarly, for right-to-left scanning, the longer were the lines, the leftward was the bisection (Table 2).

\section{Discussion}

Our results revealed that in normal subjects, irrespective of their being left-to-right or right-to-left readers, the scanning direction of a line determines the position of the subjective middle. Scanning the line from the left to the right induced a leftward deviation while scanning it from the right to the left induced a rightward deviation.

Interestingly, left neglect patients behaved qualitatively in the same way as normal subjects. Independent of their reading habits, the side of their bisection could be reversed by changing the direction of the scanning direction. Similarly to previously described cases [34], their rightward deviation is about ten times larger than the one of normal subjects in the right-to-left scanning direction. But the interesting point is that this pattern occurred toward the left when scanning from left-to-right. This finding will be discussed in the second part of the discussion.

Our findings in normal subjects confirm previous results $[9,10,12]$, emphasizing the effect of scanning direc- 
tion on active line bisection and line extension. If an imposed scanning direction may influence the perception of the subjective middle in line bisection, as it is the case in the present study, one can imagine that strong acquired directional trends like reading habits play an important role in shaping the visual space. These results confirm also previous findings concerning the effect of motor direction in straight-ahead pointing $[8,13]$.

Using a line bisection task in left neglect patients, Halligan and Marshall [19] found a leftward deviation for the smallest lines, whatever the starting position of the hand. Our results showing a systematic leftward bisection for the $5-\mathrm{cm}$ line, in the right-to-left scanning condition and even more so in the left-to-right scanning condition, are consistent with these data. It remains unclear why this phenomenon does not appear in normal subjects, but only in neglect patients. The present results underline the fact that when the hand motor component is minimized in line bisection, the position of the subjective middle is dependent upon the scanning direction. In fact, these results are quite similar to those of Halligan et al. [18], who showed that in line bisection, the right starting position of the hand (and in this way a right-to-left approach to the middle), is likely to produce a right neglect behavior (i.e., a rightward bisection), while a left starting position of the hand, and in this way a left-to-right scanning direction of the line, is likely to produce a right neglect behavior (i.e., a leftward bisection). It must be noticed that here too, the two left neglect patients did not show an improvement of their bisection when scanning the line from left-to-right instead of scanning it from right-to-left, but, as in normal subjects, opposite exploratory strategies produced opposite patterns of line bisection. It is tempting to relate the above mentioned results and the present ones to the recent paper of Bisiach et al. [3]. These authors discussed the positive effect of opto-kinetic stimulation (OKS) on left neglect behaviour. It is known that an OKS such as a large moving background produces a nystagmus with a slow phase coherent with movement and a quick phase going back to the initial fixation. This reflex keeps constant the retinal image when the body moves in the external space. Several authors have shown that leftward OKS may reduce extrapersonal or personal signs of left neglect $[22,27,35]$. Bisiach et al. [3] wonder if this transient improvement is due to a temporary rectification of the representational medium or to a modulation of attentional processes within the disordered medium. This question was addressed by asking RBD patients with and without left neglect to execute a modified line bisection task during leftward or rightward OKS. When required to set the endpoints of an imaginary horizontal line of a given length on the basis of its midpoint pointed on a sheet of paper, left neglect patients most frequently misplaced endpoints leftwards (i.e., reproducing the usual rightward deviation of the subjective middle found in line bisection). When the task was executed during leftward OKS (known to temporarily improve left neglect), the disproportion increased instead of vanishing. Bisiach et al. [3] thus proposed that manipulations such as OKS may remove neglect without normalizing the representational medium itself. In a similar way, our leftto-right scanning condition induced a pathological leftward deviation of the subjective middle in neglect patients, thus reversing their left neglect behavior without reducing it. Mattingley et al. [25] also demonstrated that the left neglect patients' rightward deviation in line bisection could be reversed by left side cues, whether visible or invisible. This result, in turn, suggests that the effect of cuing procedures (see Ref. [30]) could be explained by the scanning pattern induced by the side of the cue, which could become the starting point of the visual exploration of the stimulus.

Following this line of reasoning, the scanning direction in line bisection might interact with overt and/or covert orienting of attention [28], which are able to act upon spatial bias in neglect. This is to say that scanning direction might be crucial in determining the amount of neglect signs in brain-damaged patients. In addition it has to be noted that no current hypothesis of neglect is up to explain this effect of scanning direction on neglect signs. Our results suggest that these scanning-related effects are not specific to neglect patients but can also determine the perceptual organization of space in normal subjects. Further studies on normals and brain-damaged patients with opposite reading habits are needed to disentangle the effect of hemispheric laterality from those of environmental factors on the occurrence of lateral biases in space.

\section{Acknowledgements}

This research was supported by a grant of the RhôneAlpes Regional Council to the first author. Many thanks to $\mathrm{K}$. Benichou for her participation to this study.

\section{References}

[1] F. Abed, Cultural influences on visual scanning patterns, J. CrossCultural Psychol. 22 (1991) 525-534.

[2] P. Bartolomeo, P. D'Erme, G. Gainotti, The relationship between visuospatial and representational neglect, Neurology 44 (1994) 1710-1714.

[3] E. Bisiach, L. Pizzamiglio, D. Nico, G. Antonucci, Beyond unilateral neglect, Brain 119 (1996) 851-857.

[4] E. Bisiach, M.L. Rusconi, V.A. Peretti, G. Vallar, Challenging current accounts of unilateral neglect, Neuropsychologia 32 (1994) $1431-1434$.

[5] D. Bowers, K.M. Heilman, Pseudoneglect: effects of hemispace on a tactile line bisection task, Neuropsychologia 18 (1980) 491-498.

[6] J.L. Bradshaw, N.C. Nettleton, G. Nathan, L. Wilson, Bisecting rods and lines: effects of horizontal and vertical posture on left side underestimation by normal subjects, Neuropsychologia 23 (1985) $421-425$.

[7] J.L. Bradshaw, N.C. Nettleton, J.M. Pierson, L. Wilson, G. Nathan, Coordinates of extracorporeal space, in: M. Jeannerod (Ed.), Neuro- 
physiological and Neuropsychological Aspects of Spatial Neglect, Vol. 45, Elsevier, Amsterdam (1987) pp. 41-67.

[8] S. Chokron, P. Bartolomeo, Patterns of dissociation between left hemineglect and deviation of the egocentric reference, Neuropsychologia 35 (1997) 1503-1508.

[9] S. Chokron, J.M. Bernard, M. Imbert, Length representation in normal and neglect subjects with opposite reading habits studied through a line extension task, Cortex 33 (1997) 47-64.

[10] S. Chokron, M. De Agostini, Reading habits and line bisection: a developmental approach, Cogn. Brain Res. 3 (1995) 51-58.

[11] S. Chokron, M. Imbert, Egocentric reference and asymmetric perception of space, Neuropsychologia 31 (1993) 775-782.

[12] S. Chokron, M. Imbert, Influence of reading habits on line bisection, Cogn. Brain Res. 1 (1993) 219-222.

[13] S. Chokron, M. Imbert, Variations of the egocentric reference among normal subjects and a patient with unilateral neglect, Neuropsychologia 33 (1995) 703-711.

[14] E. De Renzi, Disorders of Space Elaboration and Cognition, Wiley, New York, 1982.

[15] G. Dellatolas, M. De Agostini, P. Jallon, M. Poncet, M. Rey, J. Lellouch, Mesure de la préférence manuelle par autoquestionnaire dans la population française adulte, Rev. Psychol. Appl. 38 (1988) $117-136$.

[16] G. Gainotti, P. D’Erme, P. Bartolomeo, Early orientation of attention toward the half space ipsilateral to the lesion in patients with unilateral brain damage, J. Neurol. Neurosurg. Psychiatry 54 (1991) 1082-1089.

[17] P. Halligan, L. Manning, J.C. Marshall, Hemispheric activation vs. spatio-motor cuing in visual neglect: a case study, Neuropsychologia 28 (1990) 1043-1051.

[18] P. Halligan, L. Manning, J.C. Marshall, Individual variation in line bisection: a study of four patients with right hemisphere damage and normal controls, Neuropsychologia 29 (1991) 165-176.

[19] P. Halligan, J.C. Marshall, Line bisection in visuo-spatial neglect: disproof of a conjecture, Cortex 25 (1989) 517-521.

[20] P. Halligan, J.C. Marshall, Perceptual cueing and perceptuo-motor compatibility in visuo-spatial neglect: a single case study, Cogn. Neuropsychol. 6 (1989) 423-435.

[21] K.M. Heilman, R.T. Watson, E. Valenstein, Neglect and related disorders, in: K.M. Heilman, E. Valenstein (Eds.), Clinical Neuropsychology, Oxford Univ. Press, New York (1993) pp. 279-336.

[22] H.-O. Karnath, Optokinetic stimulation influences the disturbed perception of body orientation in spatial neglect, J. Clin. Exp. Neuropsychol. 60 (1996) 217-220.
[23] L. Manning, P. Halligan, J.C. Marshall, Individual variation in line bisection: a study of normal subjects with application to the interpretation of visual neglect, Neuropsychologia 28 (1990) 647-655.

[24] J.B. Mattingley, J.L. Bradshaw, J.A. Bradshaw, Horizontal visual motion modulates focal attention in left unilateral spatial neglect, J. Neurol. Neurosurg. Psychiatry 57 (1994) 1228-1235.

[25] J.B. Mattingley, J.M. Pierson, J.L. Bradshaw, J.G. Phillips, J.A. Bradshaw, To see or not to see: the effects of visible and invisible cues on line bisection judgements in unilateral neglect, Neuropsychologia 31 (1993) 1201-1215.

[26] P. Nichelli, M. Rinaldi, Selective spatial attention and length representation in normal subjects and in patients with unilateral spatial neglect, Brain Cogn. 9 (1987) 57-67.

[27] L. Pizzamiglio, R. Frasca, C. Guariglia, C. Incoccia, G. Antonucci, Effect of optokinetic stimulation in patients with visual neglect, Cortex 26 (1990) 535-540.

[28] M.I. Posner, Orienting of attention, Q. J. Exp. Psychol. 32 (1980) $3-25$.

[29] P.A. Reuter-Lorenz, M.I. Posner, Components of neglect from right-hemisphere damage: an analysis of line bisection, Neuropsychologia 28 (1990) 327-333.

[30] J.A. Riddoch, G.W. Humphreys, The effect of cueing on unilateral neglect, Neuropsychologia 21 (1983) 589-599.

[31] I.H. Robertson, N. North, Spatio-motor cueing in unilateral left neglect: the role of hemispace, hand and motor activation, Neuropsychologia 30 (1992) 553-563.

[32] E. Sampaio, S. Chokron, Pseudoneglect and reversed pseudoneglect among left-handers and right-handers, Neuropsychologia 30 (1992) 797-805.

[33] D.J. Scarisbrick, J.R. Tweedy, G. Kulansky, Hand preference and performance on line bisection, Neuropsychologia 25 (1987) 695-699.

[34] T. Schenkenberg, D.C. Bradford, E.T. Ajax, Line bisection and unilateral visual neglect in patients with neurologic impairment, Neurology 30 (1980) 509-517.

[35] G. Vallar, C. Guariglia, L. Magnotti, L. Pizzamiglio, Optokinetic stimulation affects both vertical and horizontal deficits of position sense in unilateral neglect, Cortex 31 (1995) 669-683.

[36] S. Weintraub, M.M. Mesulam, Neglect: Hemispheric specialization, behavioral components and anatomical correlates, in: F. Boller, J. Grafman (Eds.), Handbook of Neuropsychology, Vol. 2, Elsevier, Amsterdam (1990) pp. 357-374.

[37] S. Weintraub, M.M. Mesulam, Visual hemispatial inattention: stimulus parameters and exploratory strategies, J. Neurol. Neurosurg. Psychiatry 51 (1988) 1481-1488. 